\title{
Hipermídia Adaptativa potencializando a inclusão digital
}

\author{
Mauricio Karrei \\ Universidade de Passo Fundo - Curso de Ciência da Computação \\ 122962@upf.br \\ Maurício Braganholi \\ Universidade de Passo Fundo - Curso de Ciência da Computação \\ 80998@upf.br \\ Éverton Roberto Auler \\ Universidade de Passo Fundo - Curso de Ciência da Computação \\ 119683@upf.br \\ Marco Antônio Sandini Trentin \\ Universidade de Passo Fundo - Curso de Ciência da Computação \\ trentin@upf.br \\ Ana Carolina Bertoletti De Marchi \\ Universidade de Passo Fundo - Curso de Ciência da Computação e Programa de \\ Pós-Graduação em Envelhecimento Humano \\ carolina@upf.br
}

\begin{abstract}
Resumo. Neste artigo propomos o uso da hipermídia adaptativa como potencializador na construção de interfaces inclusivas que possibilitam a personalização da comunidade virtual VIDE (VIvenciando na reDE). Trata-se de uma pesquisa bibliográfica que culminou na implementação de técnicas de adaptação de apresentação e de navegação com vistas a possibilitar uma melhor interação aos diferentes usuários da VIDE, auxiliando-os de forma dinâmica quanto à navegabilidade e à usabilidade, levando em conta suas características.
\end{abstract}

Palavras-chave: hipermídia adaptativa, inclusão digital, interfaces.

\section{Adaptive Hypermedia potentiating digital inclusion}

Abstract. In this paper we propose the use of adaptive hypermedia to enhance the inclusive interfaces building that allow customization of the virtual community VIDE (VIvenciando na reDE). It is about a bibliographic research that culminated in the implementation of presentation and navigation adaptation techniques, in order to enable a better interaction of the different users of VIDE, helping them a dynamically way as the navigability and usability, considering its features.

keywords: adaptive hypermedia, digital inclusion, interfaces

\section{Introdução}

O aumento da expectativa de vida da população, juntamente com a evolução constante das Tecnologias de Informação e Comunicação (TICs) tem causado, há algum tempo, um enorme impacto nos modos pelos quais as pessoas interagem.

Promover o "Acesso participativo e universal do cidadão brasileiro ao conhecimento" está entre os grandes desafios da pesquisa apontados pelo relatório da 
Sociedade Brasileira de Computação para o período de 2006 a 2016 (Baranauskas e De Souza, 2006). O relatório alerta, ainda, que conceber soluções inclusivas mediadas por tecnologias de informação e comunicação (TIC) passa pela compreensão das necessidades dos indivíduos no uso de ambientes computacionais, considerando questões como usabilidade, acessibilidade e inteligibilidade em soluções de interfaces.

$\mathrm{O}$ acesso universal e participativo apontado pela SBC vem ao encontro do conceito de inclusão social que, no âmbito das TICs, depende da inclusão digital. Segundo Vidotti e Vechiato (2009), propostas cujo foco é a inclusão digital possuem as TICs como ferramentas que podem viabilizar o desenvolvimento de habilidades e competências, direcionando o indivíduo para a inclusão social. O domínio das TICs possibilita a inclusão por facilitar o contato com familiares e amigos, conhecer diferentes pessoas e participar de grupos sociais. Ações como essas minimizam as possibilidades de exclusão social, preservando a oportunidade de os mais velhos continuarem a participar da sociedade, com autonomia, culminando em um envelhecimento de qualidade bem-sucedido (Lima et al. 2008).

No entanto, a inclusão digital quando abordada no âmbito do envelhecimento humano, apresenta obstáculos que interpõem o indivíduo mais velho com o uso das TICs. A própria essência da inclusão digital nos remete ao problema da exclusão, que, muitas vezes, é consequência de fatores como alterações físicas e cognitivas que dificultam a Interação Humano-Computador (IHC). Segundo Vidotti e Vechiato (2009), "entender os diferentes contextos do envelhecimento humano possibilita a reflexão de como construir interfaces digitais inclusivas, considerando as limitações, as potencialidades e as necessidades reais desse público específico”.

Neste trabalho propomos o uso da hipermídia adaptativa como potencializador na construção de interfaces inclusivas, adaptando a apresentação e o conteúdo aos usuários, com vistas a facilitar a interação e a inclusão de diferentes indivíduos. A adaptação considera características do usuário como: nível de compreensão da informação, assunto de interesse, língua, etc.

Diante deste contexto, realizamos uma pesquisa bibliográfica sobre diferentes técnicas de hipermídia adaptativa que culminou na implementação de uma comunidade virtual que possibilita a interação de diferentes grupos de usuários, sem que isso interfira na compreensão e na navegação do ambiente - denominada VIDE (VIvenciando na reDE).

A VIDE é uma comunidade que faz uso da hipermídia adaptativa para oferecer aos seus membros acesso diferenciado, tendo como base o seu próprio perfil. São oferecidos aos participantes vários recursos por meio de uma interface dinâmica, personalizada, adaptada aos objetivos pessoais, valorizando o usuário individualmente e incentivando a cooperação e a colaboração entre todos os membros.

Este artigo está estruturado em cinco seções. A seção 2 aborda conceitos importantes sobre a hipermídia adaptativa, diferentes formas de adaptação e técnicas. O modelo do usuário é apresentado na seção 3. Na seção 4 é descrita a VIDE, apresentando suas características. Por fim, as considerações finais são discutidas na seção 5.

\section{Hipermídia Adaptativa}

A Hipermídia Adaptativa (HA) é um recurso que possibilita a criação de ambientes virtuais dinâmicos. Brusilovsky (1996) define que "os Sistemas Hipermídia Adaptativos (SHA) consistem na área da ciência da computação que se ocupa do estudo e desenvolvimento de sistemas, arquiteturas, métodos e técnicas capazes de promover a 
adaptação de hiperdocumentos e hipermídia em geral às expectativas, necessidades, preferências e desejos de seus usuários".

Os Sistemas de Hipermídia Adaptativos tem como objetivo prover aos seus usuários informações de sua preferência, adequadas ao contexto, considerando as características individuais para a geração de uma interface que disponibiliza conteúdo e layout condizente com o seu perfil.

Paul De Bra et al. (2001) salientam que a principal motivação para a utilização de sistemas de hipermídia adaptativa tem a ver com a inadequação dos sites da Web que apresentam as mesmas informações, da mesma forma, para todos os usuários. Adicionalmente, se considerarmos os obstáculos que interpõem o indivíduo mais velho com o uso das TICs, a personalização do conteúdo e a forma de apresentação podem ser fatores motivadores para a interação e, consequente, para a inclusão digital, uma vez que levam em conta os diferentes objetivos e distintos graus de conhecimento.

Assim sendo, a hipermídia adaptativa pode ser útil em qualquer situação e/ou quando o sistema é destinado a pessoas com diferentes objetivos e conhecimentos, podendo ser aplicada em sistemas de informação e de ajuda online, sistemas de busca de informações, entre outros (Brusilovsky, 1994).

Para que um sistema seja considerado como hipermídia adaptativa, este deve satisfazer a três condições básicas (Palazzo, 2002):

1. ser um sistema com recursos de hipertexto ou hipermídia.

2. possuir um modelo de usuário.

3. ser capaz de adaptar o conteúdo hipermídia aos modelos de usuários existentes.

Os modelos de usuários tem a função de armazenar características das pessoas para, posteriormente, usar essas informações para a adaptação da forma com que o conteúdo é apresentado. A adaptação pode acontecer na apresentação ou na navegação.

Segundo Brusilovsky (1997), a adaptação da apresentação do conteúdo é utilizada para resolver o problema de sistemas hipermídia que são utilizados por diferentes classes de usuários, por exemplo, para um usuário leigo no assunto é possível oferecer explicações adicionais em diferentes mídias, enquanto para um usuário mais experiente, a apresentação da informação pode ser mais complexa. Já a adaptação da navegação é utilizada para prover apoio à navegação e para não permitir que os usuários fiquem perdidos no hiperespaço, ocultando ou mantendo links visíveis como, por exemplo, um link para um tutorial online pode estar presente para um usuário com perfil de iniciante e oculto a um usuário com perfil avançado.

Entre as técnicas utilizadas pela HA para a navegação adaptativa encontra-se (Palazzo, 2002): orientação direta, classificação adaptativa, ocultação adaptativa, anotação adaptativa e mapas adaptativos. Na comunidade virtual VIDE é utilizada a classificação adaptativa para promover a classificação dos grupos mais visitados, e a ocultação adaptativa, com vistas a ocultar links que não correspondem às preferências do usuário.

A técnica de classificação adaptativa ordena os links partindo do de maior relevância. Na VIDE são classificados os grupos mostrados ao usuário, partindo do grupo que possui mais visitas, permitindo que o usuário encontre-os com maior rapidez.

A técnica de ocultação adaptativa consiste em restringir o espaço de navegação do usuário, ocultando links para as páginas que não estão de acordo com o modelo do usuário. A ocultação protege o usuário, reduzindo a sobrecarga cognitiva. Na VIDE essa técnica é usada na visualização dos grupos, onde são apresentados, primeiramente, os grupos que correspondem ao modelo do usuário. Tal técnica é utilizada também na visualização das produções dos grupos. Tanto nas produções quanto nos grupos, as 
ocultações efetuadas podem ser acessadas pelo usuário, pois ficam visíveis na parte inferior da página.

Quanto às adaptações relativas à apresentação, as técnicas disponíveis são (Palazzo, 2002): texto condicional, stretchtext, fragmento variante, página variante e adaptação por frames. Na VIDE é utilizada a stretchtext para a exibição de texto de ajuda aos usuários com pouca experiência. O stretchtext é um tipo especial de hipertexto onde os links podem ser expandidos ou concentrados, permitindo apresentar ou ocultar condicionalmente porções de texto de acordo com o nível de conhecimento do usuário. Na VIDE o stretchtext é usado como forma de tooltip (moldura pop-up que abre quando o mouse está sobre o elemento), apresentando, ao usuário com o nível de experiência básico, informações para auxiliá-lo na comunidade.

\section{Modelo de Usuário}

Os sistemas de HA utilizam o Modelo de Usuário (MU) para armazenar as características de cada usuário. Com a identificação do modelo de usuário torna-se possível a adaptação de todo e qualquer tipo de hipermídia de forma personalizada (Aragão 2004).

Há várias características que podem e devem ser levadas em conta para a construção de um modelo de usuário em um sistema de hipermídia adaptativa. Na VIDE são armazenados:

- conhecimento: refere-se ao conhecimento que o usuário possui sobre o assunto. Pode ser considerada uma das características mais relevantes e importantes a serem levadas em conta para a construção do seu modelo. A evolução do conhecimento do usuário é um elemento crítico para a adaptação, e deve ser constantemente atualizado. A VIDE implementa este conceito estaticamente, através dos dados capturados por intervenção direta do usuário no formulário de cadastro. No futuro pretende-se trabalhar também de forma automática, unindo assim a captura estática inicial e a automática, permitindo que o sistema permaneça sempre atualizado ao usuário.

- experiência: a experiência do usuário refere-se, por exemplo, a sua familiaridade a estrutura de navegação no hiperespaço. Segundo Vassileva (1996), é diferente do conhecimento que o usuário possui acerca do assunto considerado. Usuários com pleno conhecimento do assunto tratado no hiperespaço podem desconhecer sua estrutura e vice-versa. Também é possível que o usuário domine perfeitamente a estrutura do hiperespaço, sem, entretanto, possuir qualquer conhecimento do assunto ali tratado. A experiência indica o quanto a estrutura do hipertexto é conhecida, bem como a facilidade de navegação por parte do usuário. No caso da VIDE, o usuário informa o seu nível de experiência de navegação, que pode ser Básico ou Avançado. Com essa informação o sistema o auxilia com textos explicativos, se for necessário.

- preferências: as preferências geralmente não podem ser identificadas ou deduzidas pelo sistema, sendo essas informadas diretamente pelo usuário por meio de feedback ou formulários. A VIDE utiliza formulários para que o usuário informe as áreas de interesse, os idiomas e os tipos de arquivos ou produções que são convenientes. São informações importantes para a usabilidade da interface, permitindo um conforto maior ao usuário e auxiliando-o em aspectos de acessibilidade no sistema.

O modelo de usuários da VIDE é criado a partir de intervenções diretas do usuário, garantindo fidelidade às preferências do mesmo. No entanto, a VIDE também 
grava, de forma automática, dados que permitem a aplicação de algumas técnicas de adaptação, como o número de visitas aos grupos. Tal registro é computando uma vez por dia para cada usuário evitando, assim, que algum grupo tenha sua soma de visitas alterada continuamente por um membro. Esse dado permite que seja aplicada a técnica de classificação adaptativa aos grupos.

Na VIDE é utilizado formulário para criar o modelo do usuário. O usuário é solicitado a informar seus dados que são armazenados em tabelas do banco de dados. Este formulário é composto de três partes:

- Parte 1: informações pessoais de cada usuário, requeridas e validadas por métodos de Jquery, garantido maior segurança e padronização a esses dados.

- Parte 2: preferências do usuário como: áreas de interesse, idiomas e formatos de arquivo. A área, subárea e assunto seguem a Tabela de Áreas do Conhecimento do CNPq. Quando o usuário clica no ícone em formato de “+” são exibidas as áreas de interesse em uma janela modal, permitindo a escolha de uma ou mais áreas, subáreas e assuntos. O idioma, preenchido por default como português, segue a mesma lógica de inserção das áreas de interesse.

- Parte 3: é solicitada a escolha dos tipos de arquivos de seu interesse que serão visualizados nas produções, podendo ser de formato texto (arquivos com extensões: txt, pdf, doc e docx), imagem (em formato: jpg, jpeg, gif e png), vídeo (mostrados em janelas com o youtube), páginas web (aparecerá os links para a visitação), e a opção "todos” para todos os tipos. O item Nível de Complexidade de Arquivos permite ao usuário classificar os materiais que deseja ver na comunidade, podendo ser de nível: i) básico, que são produções julgadas de simples compreensão e entendimento; ii) intermediário, compreendido como materiais com um grau de entendimento médio, e iii) avançado, exigem mais conhecimento dos usuários, ou seja, usam linguagens mais profundas e específicas; e iv) todos, faz com que todos os níveis sejam vistos na comunidade. A experiência de navegação diz ao sistema o quanto o usuário tem habilidades em navegar no ambiente e compreender suas informações, podendo assumir a condição: i) básico: onde o ambiente, através da técnica de stretchtext, fornecerá automaticamente ajuda nos termos e funcionalidades da comunidade, e ii) avançado: indicado para os usuários que possuem um conhecimento intermediário ou avançado. O último item é a escolaridade do usuário, que vai de ensino fundamental incompleto até ensino superior completo. Após a conclusão de informações, os dados são armazenados no banco de dados.

\section{A VIDE}

A VIDE (VIvenciando na reDE) é uma comunidade virtual cujo objetivo é promover a interação entre diferentes grupos de pessoas, permitindo a troca de informações por meio de textos, artigos, imagens, vídeos e páginas web e, consequentemente, potencializando a inclusão digital por meio da socialização, entretenimento e manutenção da autonomia. Foi projetada de forma a envolver seus membros e torná-los cúmplices da experiência vivenciada na rede, permitindo a criação e a participação em diferentes grupos de discussão online.

Os membros podem participar contribuindo com suas próprias produções, enriquecendo e ampliando a comunidade de acordo com suas preferências, oferecendo , com o uso da hipermídia adaptativa, acesso diferenciado conforme áreas de interesse e idioma de preferência, uma grande quantidade de informações publicadas pelos participantes dos grupos. 
A VIDE foi desenvolvida na linguagem de programação PHP, uma linguagem interpretada, independente de plataforma, capaz de implementar soluções web velozes e eficientes. Para o sistema de gerenciamento de banco de dados (SGBD) foi utilizado o MySql, devido a sua portabilidade, compatibilidade, desempenho e estabilidade. A VIDE foi desenvolvida de modo orientado a objetos, com o uso do padrão MVC (Model, View e Controller) e da biblioteca Smarty, com sistema de templates, deixando o sistema mais organizado e permitindo separar a lógica da comunidade e o conteúdo da apresentação. O principal objetivo de se utilizar a arquitetura MVC na VIDE é separar a lógica da aplicação (as regras da comunidade), da interface do usuário (as telas do programa) e do fluxo da aplicação (como são enviadas as mensagens dos eventos e para quem). Com esse padrão de camadas o sistema gera uma independência entre os componentes.

Ao entrar na comunidade pela primeira vez, o usuário preenche o formulário com dados de identificação, a partir do qual o sistema cria o modelo de usuário. Após, é aberta a página inicial, onde são exibidos os grupos existentes que se enquadram nas preferências pessoais identificadas no modelo do usuário. Para tanto, o sistema utiliza a técnica de ocultação, conforme ilustrado na Figura 1. No exemplo apresentado, a VIDE exibe ao usuário "I. da Silva” sugestões de grupos pertinentes ao seu modelo de usuário. O usuário em questão possui como área de interesse "Ciências da Saúde" e subárea "Enfermagem", as mesmas utilizadas para a criação do grupo "Formação de recursos humanos”. Os demais grupos são mostrados em Outros Grupos, possibilitando apenas a visualização. A técnica de ocultação adaptativa auxilia o usuário lhe mostrando automaticamente os grupos que são pertinentes ao seu modelo.

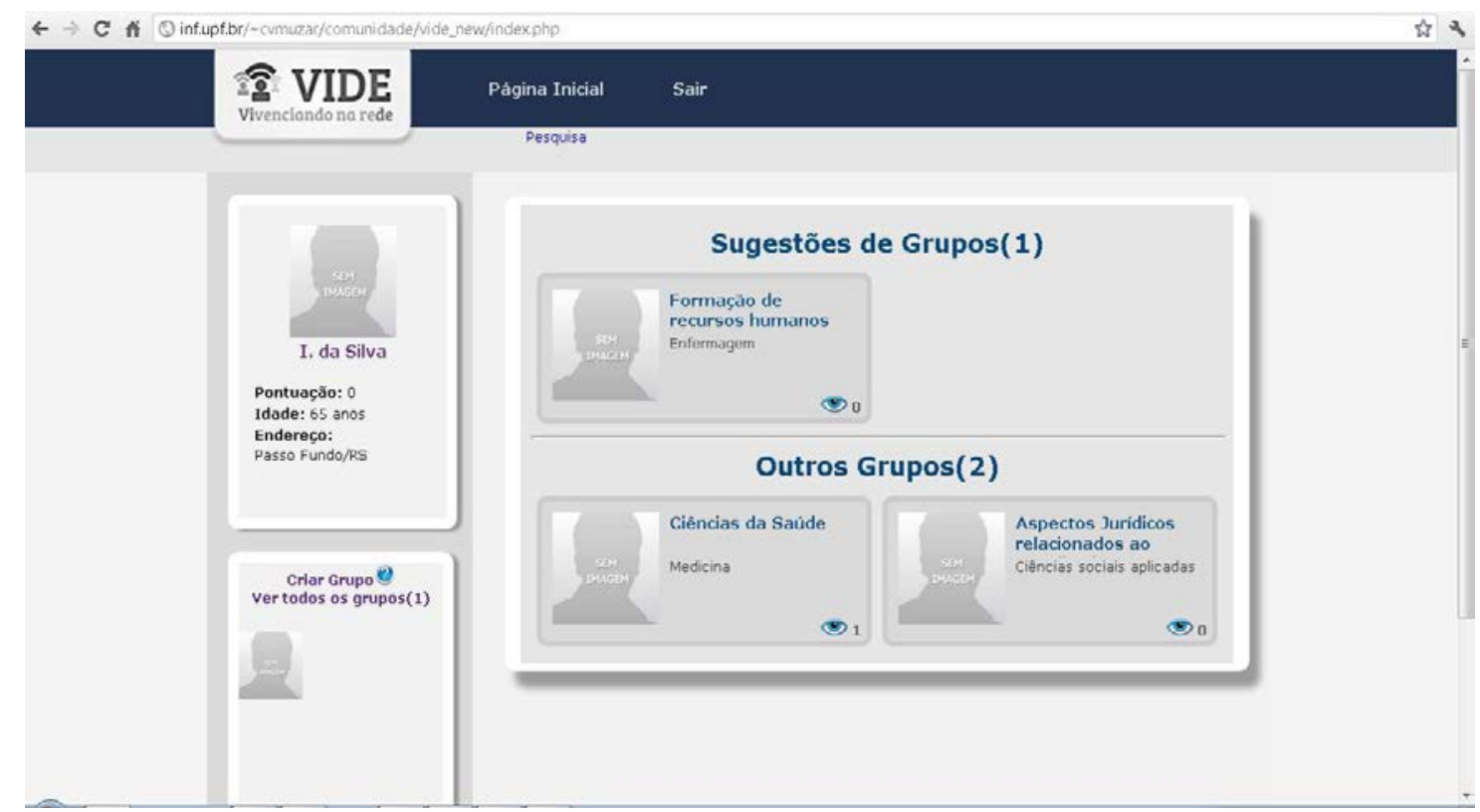

Figura 1- Exemplo da técnica de ocultação nos grupos

Para a criação de um novo grupo é preciso definir as áreas de interesse, similar ao cadastro de novos usuários, sendo necessário especificar Área (obrigatória), Subárea e Assunto. Também é preciso informar os tipos de materiais permit idos, se o grupo será do tipo público ou moderado, se possuirá chat e fórum, e quais os idiomas das produções o grupo comporta.

Quando o usuário se torna membro de um grupo, o sistema apresenta as produções deste grupo relativas ao seu modelo. Para a inserção de produções, o usuário 
deve obrigatoriamente pertencer ao grupo. Tal inserção exige que sejam informados os seguintes dados: Tipo do Material, Título, Idioma (opcional em imagens) e Complexidade. Os demais dados são opcionais: Autor, Assunto, Descrição e Data de criação. O idioma é definido pelo criador do grupo.

A Figura 2 exemplifica outra situação em que foi aplicada a técnica de ocultação adaptativa, referente às produções do grupo. O grupo "Computação PF" está sendo visitado pelo membro "Fernando E.”, que possui em seu modelo de usuário interesse pelo idioma Português e todos os tipos de arquivos. Na imagem podemos observar que o sistema só lhe mostrou como sugestão a produção "Código Genético" do tipo "Imagem", pois é a única produção que satisfaz ao modelo. A produção "Máquina Enigma”, mesmo sendo do tipo Imagem, tem como idioma o Inglês, incompatível ao modelo do usuário Fernando E. As demais produções no formato texto estão no idioma Inglês, consequentemente ficam em Outros, por não corresponderem ao modelo do usuário em questão. Da mesma forma que a ocultação dos grupos, a ocultação das produções não limita o usuário a somente ter acesso às produções fieis ao seu modelo, mostrando-as em Outros e dando-lhe a liberdade de acesso.

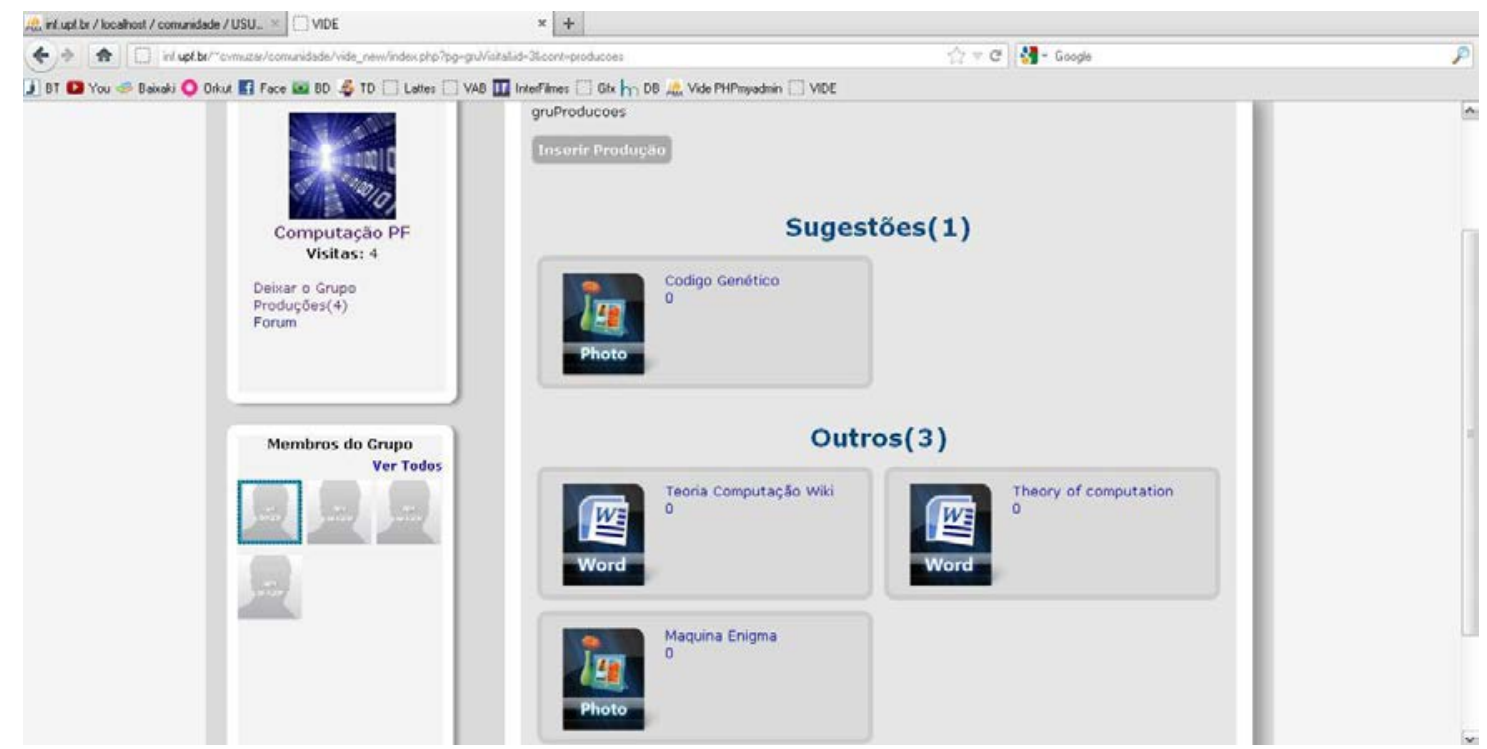

Figura 2 - Exemplo da técnica de ocultação adaptativa nas produções

A técnica de stretchtext foi aplicada na comunidade para proporcionar aos usuários, com nível de experiência de navegação Básico, o acesso a um texto explicativo, conforme ilustrado na Figura 3. Essa técnica oculta e expande o texto, estando empregado na comunidade como forma de tooltip. Desta forma, quando o usuário passar o mouse no ícone “?”, um texto de apoio é expandido, indicando para o que é, ou o que é a expressão ou item relacionado. Ao retirar o mouse do ícone, o texto se oculta. Este recurso não aparece aos usuários que possuem experiência Avançada registrada em seu modelo de usuário.

A técnica de classificação adaptativa também foi empregada na comunidade para exibir/classificar os grupos de acordo com o número de visitas. A Figura 4 exibe duas imagens da tela inicial do usuário "Mauricio B" em momentos distintos. A imagem da esquerda apresenta uma organização na visualização dos grupos, e na imagem da direita, capturada após algum tempo de uso do sistema, a organização dos grupos foi alterada, apresentando do grupo mais visitado ao menos. Desse modo, o usuário tem sempre a classificação dos grupos com mais visitas nas primeiras posições de visualização da tela. 


CINTED-UFRGS Novas Tecnologias na Educação

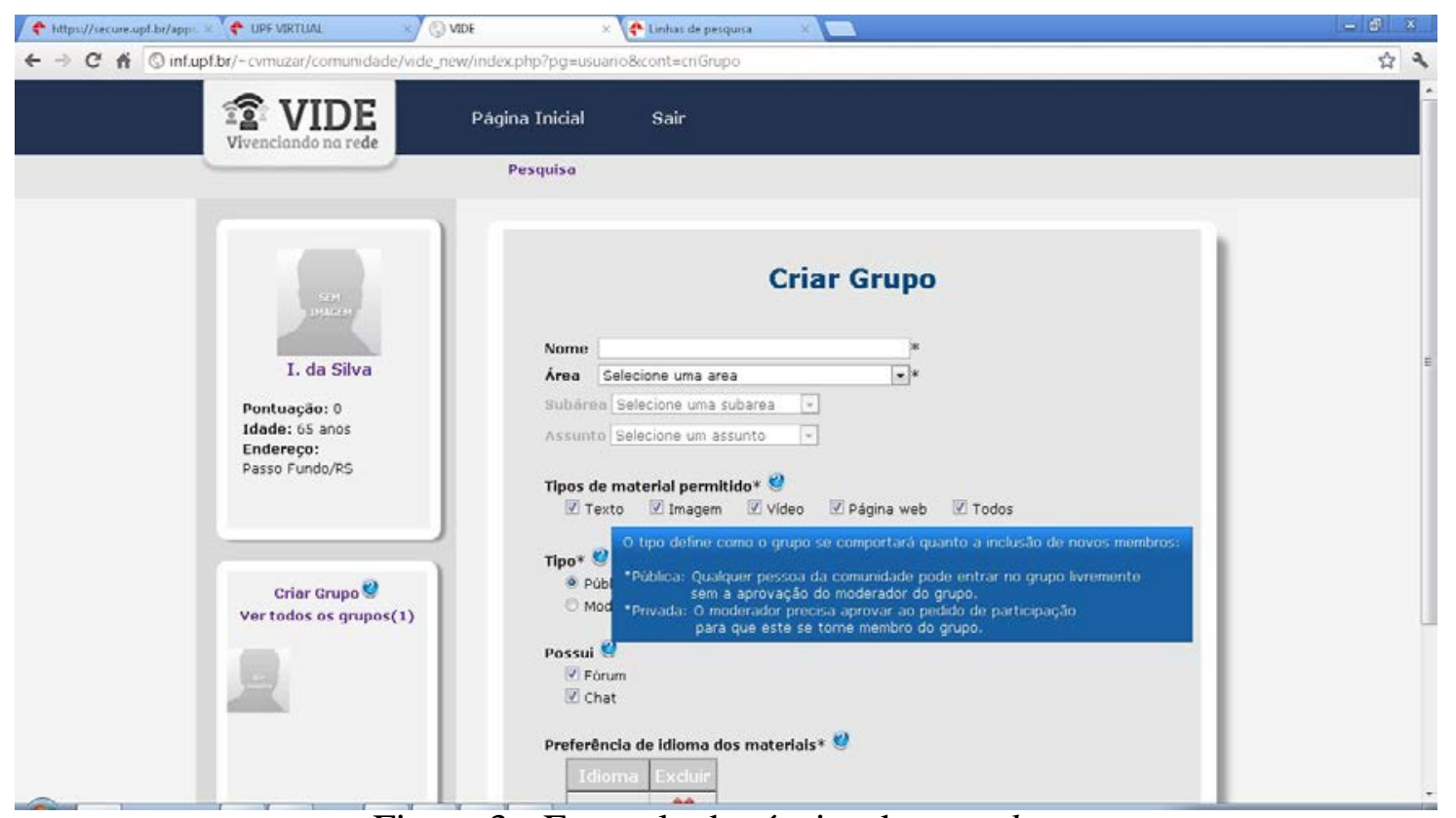

Figura 3 - Exemplo da técnica de stretchtext

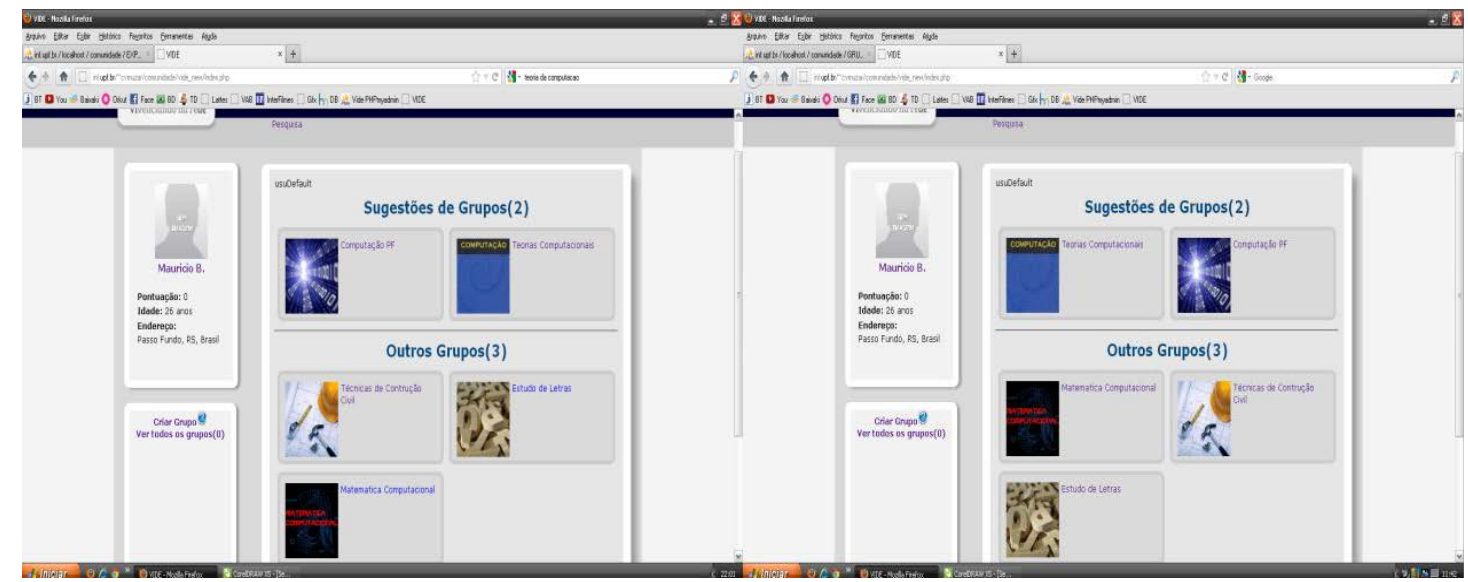

Figura 4 - Exemplo da técnica de classificação adaptativa

Com o uso das técnicas de adaptação descritas e exemplificadas acima, acreditamos que a interação humano-computador seja facilitada, beneficiando diferentes tipos de usuários e auxiliando na inclusão digital. A VIDE torna-se, com isso, um exemplo de sistema que permite o acesso universal e participativo, apontado pela SBC como desafios da Computação.

\section{Conclusão}

Nosso objetivo neste estudo foi implementar técnicas de hipermídia adaptativa na Comunidade Virtual VIDE com vistas a possibilitar a interação de diferentes grupos de usuários, sem que isso interfira na compreensão e na navegação do ambiente e auxilie na inclusão digital. Para tanto, o protótipo desenvolvido promove a adaptação da comunidade aos usuários de acordo com seus próprios perfis.

Dentre os impactos objetivados por este trabalho, o mais importante pode ser destacado como a possibilidade de melhoria na utilização e navegação de uma comunidade virtual através de técnicas de hipermídia adaptativa, promovendo assim a personalização de acordo com as preferências específicas de cada usuário, gerando, 
consequentemente, uma maior motivação dos mesmos para a aprendizagem e troca de materiais com os outros membros da comunidade.

Nossas perspectivas para trabalhos futuros são: i) identificar características dos usuários de forma automática pelo sistema, de modo a complementar o seu modelo de usuário com informações capturadas durante a navegação na comunidade e ii) validar o protótipo de modo a identificar fatores limitantes e melhor explorar fatores que potencializem a utilização de comunidades virtuais apoiadas por sistemas de hipermídia adaptativa.

\section{Referências Bibliográficas}

ARAGÃO, A. L. (2004). Utilização de aprendizado de máquina para adaptação de estruturas em hipermídia adaptativa. São Paulo, 2004. Dissertação (Mestrado pelo Instituto de Ciências Matemáticas e da Computação, da USP), 92p.

BARANAUSKAS, M. C. C.; DE SOUZA, C. S. (2006) Desafio 4: Acesso participativo e universal do cidadão brasileiro ao conhecimento. Computação Brasil, Porto Alegre, v. 23, p. 7-7.

BRUSILOVSKY, P. (1994). Adaptive hypermedia: the State of the Art. In: Proceedings of the East-West International Conference on Multimedia, Hypermedia, and Virtual Reality - MHVR'94.

BRUSILOVSKY, P. (1996). Methods and Techniques of Adaptive Hypermedia. User Modeling and User-Adapted Interaction. Special issue on adaptive hypertext and hypermedia, Dordrecht, v.6, n.2-3, p.87-129.

BRUSILOVSKY, P. (1997). Efficient Techniques for Adaptive Hypermedia. Intelligent hypertext: Advanced techniques for the World Wide Web. Lectures Notes in Computer Science, v. 1326, Berlin, Spring-Verlag, p. 12-30.

DE BRA, P.; AERTS, A.; HOUBEN, G.; WU, H. (2001) Making General-Purpose Adaptive Hypermedia Work. In: World Conference on the WWW and Internet, 2001. Orlando, EUA. Anais. Orlando: Association for the Advancement of Computing in Education, 2001, p.117-123.

LIMA, A.M.M.; SILVA, H.S.; GALHARDONI, R. (2008) Successful aging: paths for a construct and new frontiers. Interface - Comunic., Saúde, Educ., v.12, n.27, p.795807.

PALAZZO,L.A.M. (2002). Sistemas de hipermídia adaptativa. Caderno de publicações da SBC.

VASSILEVA, J. (1996) A task-centered approach for user modelling in a hypermedia office documentation system. User Modeling and User-Adapted Interaction. Special issue on adaptive hypertext and hypermedia, Dordrecht, v.6, n.2-3, p.87-129.

VIDOTTI, S. A. B. G. e VECHIATO, F. (2009). Aspectos biológicos e psicossociais do envelhecimento humano e suas implicações no desenvolvimento de interfaces digitais: contribuição para a inclusão digital de idosos. In: IV Congreso de La CiberSociedad. 